\title{
Correct definition of color singlet P-wave non-perturbative matrix element of heavy quarkonium production
}

\author{
Gouranga C. Nayak ${ }^{1}$ \\ C.N. Yang Institute for Theoretical Physics, Stony Brook University, \\ Stony Brook NY, 11794-3840 U.S.A. \\ E-mail: nayakg138@gmail.com
}

ABSTRACT: Recently we have proved factorization of infrared divergences in NRQCD Swave heavy quarkonium production at high energy colliders at all orders in coupling constant. One of the problem which still exists in the higher order pQCD calculation of color singlet $\mathrm{P}$-wave heavy quarkonium production/anihillation is the appearance of noncanceling infrared divergences due to real soft gluons exchange, although no such infrared divergences are present in the color singlet S-wave heavy quarkonium. In this paper we find that since the non-perturbative matrix element of the color singlet $\mathrm{P}$-wave heavy quarkonium production contains derivative operators, the gauge links are necessary to make it gauge invariant and be consistent with the factorization of such non-canceling infrared divergences at all orders in coupling constant.

KeYwords: Phenomenological Models, QCD Phenomenology

ArXIV EPRINT: 1704.07449

\footnotetext{
${ }^{1}$ G.C. Nayak was affiliated with C.N. Yang Institute for Theoretical Physics in 2004-2007.
} 


\section{Contents}

1 Introduction

2 Infrared divergence in higher order pQCD calculation in color singlet P-wave heavy quarkonium

3 Lack of gauge invariance in NRQCD S-wave color octet non-perturbative matrix element

4 Infrared divergence due to real soft gluon (eikonal gluon) emission/absorption in QCD

5 Proof of factorization of infrared divergence in color singlet P-wave heavy quarkonium production

6 Correct definition of non-perturbative matrix element of color singlet $\mathrm{P}$-wave heavy quarkonium production

\section{Introduction}

Recently we have proved factorization of infrared divergences in NRQCD S-wave heavy quarkonium production at high energy colliders at all orders in coupling constant in [1]. In this paper we extend this to prove factorization of infrared divergences in color singlet P-wave heavy quarkonium production at high energy colliders at all orders in coupling constant. We predict the correct definition of the color singlet P-wave non-perturbative matrix element of heavy quarkonium production.

Since the discovery of $j / \psi[2,3]$ there have been lot of experimental progress in the measurement of heavy quarkonium production/decay both at fixed target experiments and at collider experiments. On the theoretical side the understanding of heavy quarkonium production/decay proceeds in two steps, 1) the production/anihilation of heavy quarkantiquark pair which can be calculated by using pQCD [4-7] and 2) the formation of heavy quarkonium from heavy quark-antiquark pair which involves non-perturbative QCD which is not solved yet. Hence, unlike QED, the non-perturbative matrix element of the heavy quarkonium in $\mathrm{QCD}$ is extracted from the experiment.

Assuming that the factorization theorem holds in QCD, the heavy quarkonium production from color singlet heavy quark-antiquark pair is given by

$$
d \sigma_{p p \rightarrow H+X\left(P_{T}\right)}=\sum_{i, j} \int d x_{1} d x_{2} f_{i / p}\left(x_{1}, Q\right) f_{j / p}\left(x_{2}, Q\right) d \hat{\sigma}_{i j \rightarrow Q \bar{Q}\left[{ }^{2 S+1} L_{J}\right]+X\left(P_{T}\right)}<0\left|\mathcal{O}_{H}\right| 0>
$$


where the orbital angular momentum quantum number $L=0$ corresponds to S-wave heavy quarkonium such as $\eta_{c}, j / \psi$ and $L=1$ corresponds to P-wave heavy quarkonium such as $h_{c}, \chi_{J}$ where $S$ and $J$ are the spin and total angular momentum quantum numbers of the heavy quarkonium. The partonic level cross section for color singlet heavy quark-antiquark $(Q \bar{Q})$ pair production is given by $d \hat{\sigma}_{i j \rightarrow Q \bar{Q}\left[{ }^{2 S+1} L_{J}\right]+X\left(P_{T}\right)}$ which can be calculated by using pQCD where $i, j=q, \bar{q}, g$. The $f_{i / p}(x, Q)$ is the parton distribution function of the parton $i$ inside the hadron $p$. The non-perturbative matrix element of the heavy quarkonium $(H)$ is given by $<0\left|\mathcal{O}_{H}\right| 0>$. The heavy quarkonium is also denoted by $J^{P C}$ where $P=(-1)^{L+1}$ and $C=(-1)^{L+S}$.

Since the partonic level cross section $d \hat{\sigma}_{i j \rightarrow Q \bar{Q}\left[{ }^{2 S+1} L_{J}\right]+X\left(P_{T}\right)}$ is calculated by using pQCD one expects to encounter non-canceling infrared divergences when pQCD calculation is performed at higher orders in the QCD coupling constant. There are two types of infrared divergence in this case, 1) infrared divergence due to the Coulomb gluon exchange and 2) infrared divergence due to real soft gluon emission/absorption (eikonal gluon) [8].

For S-wave and P-wave heavy quarkonium the appearance of infrared divergence due to Coulomb gluon exchange (which is also known as $\frac{1}{v}$ divergence [9-14] in the limit $v \rightarrow 0$ where $v$ is the relative velocity between heavy quark and antiquark) is usually handled by normalization of the bound state wave function similar to QED [15] where the Coulomb potential is used.

However, one may expect non-canceling infrared divergence due to emission/absorption of real soft gluon (eikonal gluon). In the higher order pQCD calculation of the color singlet S-wave heavy quark-antiquark production/anihillation one finds the absence of such infrared divergence $[9,16]$. Hence the non-perturbative effects can be factored into the nonperturbative wave function at the origin $R(0)$ for the S-wave heavy quarkonium. In terms of heavy quark fields one finds that the definition of the gauge invariant non-relativistic wave function at the origin of the color singlet S-wave heavy quarkonium is given by [17]

$$
|R(0)|^{2}=\frac{2 \pi}{3}<0\left|\mathcal{O}_{H}\right| 0>=\frac{2 \pi}{3}<0\left|\chi^{\dagger} \xi a_{H}^{\dagger} a_{H} \xi^{\dagger} \chi\right| 0>
$$

where $\chi$ is the two component Dirac spinor field that creates a heavy quark and $\xi$ is the two component Dirac spinor field that annihilates a heavy quark and $a_{H}^{\dagger}$ is the creation operator of the heavy quarkonium $H$. In eq. (1.2) the non-perturbative matrix element $<0\left|\chi^{\dagger} \xi a_{H}^{\dagger} a_{H} \xi^{\dagger} \chi\right| 0>$ is evaluated at the origin.

However, unlike higher order pQCD calculation of color singlet S-wave heavy quarkantiquark production/anihillation, the higher order pQCD calculation of color singlet Pwave heavy quark-antiquark production/anihillation contains non-canceling infrared divergence due to real soft gluons emission/absorption [10-14]. Hence the non-perturbative effects can not be factored into the derivative of the non-relativistic wave function at the origin [17]

$$
\left|R^{\prime}(0)\right|^{2}=\frac{2 \pi}{27}<0\left|\mathcal{O}_{H}\right| 0>=\frac{2 \pi}{27}<0\left|\chi^{\dagger} \bar{\nabla} \xi a_{H}^{\dagger} \cdot a_{H} \xi^{\dagger} \bar{\nabla} \chi\right| 0>
$$

where

$$
\chi^{\dagger} \bar{\nabla} \xi=\chi^{\dagger}(\vec{\nabla} \xi)-(\vec{\nabla} \chi)^{\dagger} \xi
$$


and the non-perturbative matrix element $<0\left|\chi^{\dagger} \bar{\nabla} \xi a_{H}^{\dagger} \cdot a_{H} \xi^{\dagger} \bar{\nabla} \chi\right| 0>$ is evaluated at the origin.

This is also easy to see from eq. (1.3) because, unlike $|R(0)|^{2}$ in eq. (1.2) which is gauge invariant, the $\left|R^{\prime}(0)\right|^{2}$ in eq. (1.3) is not gauge invariant. Since the issue of gauge invariance and non-canceling infrared divergences due to real soft gluons emission/absorption are related [18-20], one expects that the gauge links need to be supplied in eq. (1.3) to make $\left|R^{\prime}(0)\right|^{2}$ gauge invariant and consistent with the factorization of such non-canceling infrared divergences due to the real soft gluons emission/absorption. In this paper we will investigate this in detail.

We find that the correct definition of the gauge invariant non-perturbative matrix element of the color singlet $\mathrm{P}$-wave heavy quarkonium production which is consistent with factorization of infrared divergences is given by

$$
<0\left|\mathcal{O}_{H}\right| 0>=<0\left|\chi^{\dagger} \Phi \bar{\nabla} \Phi^{\dagger} \xi a_{H}^{\dagger} \cdot a_{H} \xi^{\dagger} \Phi \bar{\nabla} \Phi^{\dagger} \chi\right| 0>
$$

where

$$
\Phi(x)=\mathcal{P} e^{-i g T^{c} \int_{0}^{\infty} d \lambda l \cdot A^{c}(x+\lambda l)}
$$

is the gauge link in the fundamental representation of $\mathrm{SU}(3)$ and

$$
\chi^{\dagger} \Phi \bar{\nabla} \Phi^{\dagger} \xi=\chi^{\dagger} \Phi\left(\vec{\nabla} \Phi^{\dagger} \xi\right)-\left(\vec{\nabla} \Phi^{\dagger} \chi\right)^{\dagger} \Phi^{\dagger} \xi
$$

We find that the long-distance behavior of the color singlet $\mathrm{P}$-wave non-perturbative matrix element $<0\left|\chi^{\dagger} \Phi \bar{\nabla} \Phi^{\dagger} \xi a_{H}^{\dagger} \cdot a_{H} \xi^{\dagger} \Phi \bar{\nabla} \Phi^{\dagger} \chi\right| 0>$ in eq. (1.5) is independent of the lightlike vector $l^{\mu}$ at all order in coupling constant in QCD where $l^{\mu}$ is used to define the gauge link in eq. (1.6).

Note that there is no gauge link in $|R(0)|^{2}$ in eq. (1.2) in case of S-wave heavy quarkonium because there are no derivative operators like that in eq. (1.5) for P-wave heavy quarkonium. The $|R(0)|^{2}$ in eq. (1.2) is gauge invariant without gauge links which is consistent with the fact that there are no uncanceled infrared divergences due to real soft gluons emission/absorption in case of color singlet S-wave heavy quarkonium.

In this paper we will provide a derivation of eq. (1.5).

The paper is organized as follows. In section 2 we briefly review the appearance of infrared divergences in higher order pQCD calculation in color singlet P-wave heavy quarkonium. In section 3 we discuss the lack of gauge invariance in S-wave NRQCD color octet non-perturbative matrix element which is used to address the issue of noncanceling infrared divergences in the color singlet P-wave heavy quarkonium production in the literature. In section 4 we describe the infrared divergence due to real soft gluon (eikonal gluon) emission/absorption in pQCD. In section 5 we prove the factorization of infrared divergences in color singlet $\mathrm{P}$-wave heavy quarkonium production at all orders in coupling constant at high energy colliders. In section 6 we derive the correct definition of the gauge invariant non-perturbative matrix element of the color singlet P-wave heavy quarkonium production as given by eq. (1.5). Section 7 contains conclusions. 


\section{Infrared divergence in higher order pQCD calculation in color singlet P-wave heavy quarkonium}

In the higher order pQCD calculation of heavy quark-antiquark annihilation in the color singlet $\mathrm{P}$-wave state, the non-canceling infrared divergences were found for the color singlet P-wave heavy quarkonium decay to hadrons [10-14]. Similarly in the higher order pQCD calculation of hadroproduction of heavy quark-antiquark in the color singlet P-wave state, the non-canceling infrared divergences were found [8]. A satisfactory explanation of these non-canceling infrared divergences in the case of color singlet P-wave heavy quarkonium production/decay has not been given in the literature, partly because of the lack of understanding of the correct definition of the gauge invariant non-perturbative matrix element of the color singlet P-wave heavy quarkonium which is required to cancel these infrared divergences (factorization).

In addition to this it should be noted that the diagrammatic pQCD calculation is not always sufficient to predict the correct definition of non-perturbative matrix element in QCD, no matter how many orders of pQCD calculation is performed. On the other hand the non-perturbative QCD method (the path integral formulation of QCD) can correctly predict the correct definition of the non-perturbative matrix element in QCD. We will use path integral formulation of QCD in this paper.

In case of $h_{c}$ decays to hadrons the partonic process in which the non-canceling infrared divergence is found is given by [21]

$$
{ }^{1} P_{1} \rightarrow g g g
$$

Similarly in case of $\chi_{c J}$ decay to hadrons the partonic process in which the non-canceling infrared divergence is found is given by $[10-14]$

$$
{ }^{3} P_{J} \rightarrow q \bar{q} g .
$$

Note that the collinear divergences are canceled by the virtual corrections to the two gluon decay process [8]. Hence the non-canceling infrared divergence is due to the emission/absorption of real soft gluon (eikonal gluon).

Similar to the hadronic decay of color singlet P-wave heavy quarkonium, the noncanceling infrared divergences are found in the hadroproduction of color singlet P-wave heavy quarkonium. For example, the non-canceling infrared divergence appears in the partonic process [8]

$$
q \bar{q} \rightarrow{ }^{3} P_{J} g .
$$

Note that unlike decay process in eq. (2.2) there is no collinear divergence in the production process in eq. (2.3) and hence there is no virtual correction present either. The non-canceling infrared divergence is due to emission/absorption of real soft gluon (eikonal gluon).

It can be noted that in the original calculation [10-14] the binding energy was used as infrared cutoff which can be mapped into the corresponding infrared divergence analysis in the dimensional regularization scheme, see for example [22]. 
Hence whether it is color singlet P-wave heavy quarkonium production or decay, the non-canceling infrared divergences occur due to real soft gluons emission/absorption. In this paper we will derive the correct definition of the gauge invariant color singlet $\mathrm{P}$-wave non-perturbative matrix element which cancels these infrared divergences at all order in coupling constant in QCD.

We emphasize here that we are not calculating the finite part of the cross section of the color singlet $\mathrm{P}$-wave heavy quarkonium production in the partonic level processes like that in eq. (2.3). What we are investigating in this paper is to study the exact behavior of the infrared divergences due to real soft gluons emission/absorption without modifying the finite value of the cross section. Our main aim is to derive the correct definition of the gauge invariant color singlet $\mathrm{P}$-wave non-perturbative matrix element which cancels these infrared divergences at all orders in coupling constant.

The color singlet $\mathrm{P}$-wave non-perturbative matrix element which was used in the earlier studies is given by eq. (1.3) which is not gauge invariant. Hence it is no surprise that the non-canceling infrared divergences were present in these earlier studies of color singlet $\mathrm{P}$-wave heavy quarkonium production/decay. It is expected that if the gauge links are supplied in the definition of the non-perturbative matrix element to make it gauge invariant then it can accommodate these non-canceling infrared divergences [18-20]. Hence when we use the gauge invariant definition of the color singlet $\mathrm{P}$-wave non-perturbative matrix element as given by eq. (1.5) then we do not expect any uncanceled infrared divergences due to real soft gluons emission/absorption in the color singlet P-wave heavy quarkonium production.

It is useful to mention here that the heavy quarkonium is also an useful signature [23] to study production and detection of quark-gluon plasma at RHIC and LHC. In high energy heavy-ion collisions at RHIC and LHC the study of heavy quarkonium production is more complicated than that in $p p$ collisions due to the presence of $\mathrm{QCD}$ medium in non-equilibrium.

\section{Lack of gauge invariance in NRQCD S-wave color octet non-pertur- bative matrix element}

It can be mentioned here that in the NRQCD approach of heavy quarkonium study the S-wave color octet non-perturbative matrix element

$$
<0\left|\mathcal{O}_{H}\right| 0>=<0\left|\chi^{\dagger} T^{a} \xi a_{H}^{\dagger} a_{H} \xi^{\dagger} T^{a} \chi\right| 0>
$$

is used to factorize the above mentioned non-canceling infrared divergences which arise due to real soft gluons emission/absorption (eikonal gluons) in the pQCD partonic level calculation in the color singlet P-wave heavy quarkonium production [17]. The NRQCD definition of the color singlet $\mathrm{P}$-wave non-perturbative matrix element used for this purpose is given by [17]

$$
<0\left|\mathcal{O}_{H}\right| 0>=<0\left|\chi^{\dagger} \overline{\mathbf{D}} \xi a_{H}^{\dagger} \cdot a_{H} \xi^{\dagger} \overline{\mathbf{D}} \chi\right| 0>
$$

which is gauge invariant where $\mathbf{D}$ is the covariant derivative. 
Note that in QCD the definition of the color singlet P-wave non-perturbative matrix element of the heavy quarkonium is given by eq. (1.3) which contains the ordinary derivative $\nabla$ whereas in NRQCD the definition of the color singlet P-wave non-perturbative matrix element of the heavy quarkonium is given by eq. (3.2) which contains covariant derivative D. The main motivation to use covariant derivative $\mathbf{D}$ in NRQCD instead of ordinary derivative $\nabla$ was to make the the definition in eq. (3.2) gauge invariant. However, it has to be remembered that it is the relative momentum q of the heavy quark-antiquark pair which appears in the definition of the color singlet P-wave non-prturbative matrix element [10-14] which can be obtained if ordinary derivative $\nabla$ operates on the heavy quark field which can be seen from the definition in eq. (1.3) in QCD.

Of course the appearance of ordinary derivative $\nabla$ makes the definition of the color singlet $\mathrm{P}$-wave non-perturbative matrix element of heavy quarkonium in eq. (1.3) gauge non-invariant which is the reason why we have non-canceling infrared divergences because the non-canceling infrared divergences due to real soft gluons (eikonal gluons) emission/absorption can not be absorbed into the gauge non-invariant definition of the color singlet P-wave non-perturbative matrix element of heavy quarkonium in eq. (1.3). When gauge links are present in the gauge invariant definition of the color singlet Pwave non-perturbative matrix element of heavy quarkonium as given by eq. (1.5) then the non-canceling infrared divergences due to the real soft gluons (eikonal gluons) emission/absorption can be absorbed into this gauge invariant definition of the color singlet P-wave non-perturbative matrix element of heavy quarkonium in eq. (1.5). Hence, unlike NRQCD which brings color octet mechanism to solve infrared divergences problem in color singlet mechanism, we do not bring color octet mechanism to solve infrared divergences problem in color singlet mechanism.

In any case let us briefly discuss the issue of lack of gauge invariance of the S-wave color octet non-perturbative matrix element of the heavy quarkonium production in NRQCD. In order for the non-canceling infrared divergences due to real soft gluons (eikonal gluons) emission/absorption in the color singlet $\mathrm{P}$-wave heavy quarkonium production to be factorized into the definition of the NRQCD S-wave color octet non-perturbative matrix element as given by eq. (3.1), the two non-perturbative matrix elements in NRQCD in eqs. (3.1) and (3.2) are related by the equation (see eq. (6.5) of [17])

$$
\Lambda \frac{d}{d \Lambda}<0\left|\chi^{\dagger} T^{a} \xi a_{H}^{\dagger} a_{H} \xi^{\dagger} T^{a} \chi\right| 0>=\frac{4 C_{F} \alpha_{s}(\Lambda)}{3 N_{c} \pi M^{2}}<0\left|\chi^{\dagger} \overline{\mathbf{D}} \xi a_{H}^{\dagger} \cdot a_{H} \xi^{\dagger} \overline{\mathbf{D}} \chi\right| 0>
$$

which has the problem of gauge invariance in NRQCD because the left hand side of eq. (3.3) is not gauge invariant whereas the right hand side of eq. (3.3) is gauge invariant where $\Lambda$ is the ultraviolet cutoff of NRQCD. This is because the definition of the NRQCD S-wave color octet non-perturbative matrix element as given by eq. (3.1) in NRQCD in [17] is not gauge invariant. Note that the gauge links are supplied in the NRQCD S-wave color octet non-perturbative matrix element in $[1,18-20]$ to make it gauge invariant and consistent with factorization of infrared divergences.

It can be mentioned here that in NRQCD, the (the long distance matrix elements) LDMEs are organized as powers of the relative velocity $(v)$ of the heavy quarks in the 
heavy-quarkonium states. At leading order in $v^{2},\left|R^{\prime}(0)\right|^{2}$ in eq. (1.3), which is usually calculated in potential models in Coulomb gauge, is gauge invariant and so is the evolution equation eq. (3.3).

\section{Infrared divergence due to real soft gluon (eikonal gluon) emission/ absorption in QCD}

As mentioned in section 2 the non-canceling infrared divergences occur in the color singlet P-wave heavy quarkonium production in the processes like that in eq. (2.3) due to real soft gluons (eikonal gluons) emission/absorption. Hence in this section we will describe the infrared divergence due to real soft gluon (eikonal) emission/absorption in QCD. Before describing infrared divergence due to soft gluon (eikonal gluon) emission/absorption in QCD let us first describe the infrared divergence due to soft photon (eikonal photon) emission/absorption in QED.

Consider the emission of a real photon with four momentum $k^{\mu}$ from an electron of four momentum $p^{\mu}$ in QED. The Feynman diagram contribution from this real photon emission process in QED is given by [24]

$$
\frac{1}{\not p-\not k-m} \not(k) u(p)=-\frac{\not p-\not k+m}{2 p \cdot k} \not(k) u(p)=-\frac{p \cdot \epsilon(k)}{p \cdot k} u(p)+\frac{\not k \notin(k)}{2 p \cdot k} u(p)
$$

where we have used

$$
(\not p-m) u(p)=0 .
$$

The photon field $\epsilon^{\mu}(k)$ can be written as

$$
\epsilon^{\mu}(k)=\left[\epsilon^{\mu}(k)-\frac{k^{\mu}}{p \cdot k} p \cdot \epsilon(k)\right]+\frac{k^{\mu}}{p \cdot k} p \cdot \epsilon(k)=\epsilon_{\text {phys }}^{\mu}(k)+\epsilon_{\text {pure }}^{\mu}(k)
$$

where $\epsilon_{\text {phys }}^{\mu}(k)$ is the transversely polarized (physical) photon field and $\epsilon_{\text {pure }}^{\mu}(k)=\frac{k^{\mu}}{p \cdot k} p \cdot \epsilon(k)$ is the (unphysical) longitudinally polarized pure gauge photon field.

Hence we expect that it is the transversely polarized (physical) photon field $\epsilon_{\text {phys }}^{\mu}(k)$ in eq. (4.3) that contributes to the physical (finite) value of the cross section but the (unphysical) longitudinally polarized pure gauge photon field $\epsilon_{\text {pure }}^{\mu}(k)$ does not contribute to the physical (finite) value of the cross section. This can be seen by using eq. (4.3) in (4.1) to find

$$
\frac{k k(k)}{2 p \cdot k}=\frac{k k k_{\text {phys }}(k)}{2 p \cdot k} \rightarrow \text { finite } \quad \text { when } \quad k^{\mu} \rightarrow 0
$$

and

$$
\frac{\not k k_{\text {pure }}(k)}{2 p \cdot k}=0
$$

Eq. (4.4) is the non-eikonal contribution from this soft photon emission Feynman diagram which contributes to the finite value of the physical cross section whereas from eq. (4.5) we 
find that the (unphysical) longitudinally polarized pure gauge photon field $\epsilon_{\text {pure }}^{\mu}(k)$ can not contribute to the finite value of the physical cross section. The (unphysical) longitudinally polarized pure gauge photon field $\epsilon_{\text {pure }}^{\mu}(k)$ in eq. (4.3) accounts for the infrared divergence from this soft photon emission Feynman diagram. This can be seen as follows.

By using eq. (4.3) in (4.1) we find

$$
\frac{p \cdot \epsilon(k)}{p \cdot k}=\frac{p \cdot \epsilon_{\text {pure }}(k)}{p \cdot k} \rightarrow \infty \quad \text { when } \quad k^{\mu} \rightarrow 0 .
$$

and

$$
\frac{p \cdot \epsilon_{\mathrm{phys}}(k)}{p \cdot k}=0
$$

Eq. (4.6) gives the infrared divergence in the eikonal approximation from this real soft photon (eikonal photon) emission Feynman diagram. From eq. (4.7) we find that the transversely polarized (physical) photon field $\epsilon_{\text {phys }}^{\mu}(k)$ in eq. (4.3) does not contribute to the infrared divergence from this real soft photon emission Feynman diagram in the eikonal approximation.

Hence from eqs. (4.6), (4.5) and (4.1) we find that if the charge produces pure gauge field then the study of infrared divergence due to soft photon (eikonal photon) emission from that charge can be simplified in quantum field theory by using pure gauge.

In our calculation the momentum $P_{1}^{\mu}$ of the heavy quark can be different from the momentum $P_{2}^{\mu}$ of the heavy antiquark where

$$
\vec{P}_{1}-\vec{P}_{2}=2 m \vec{v} .
$$

If the photon interacts with the electron and positron simultaneously then that photon is the virtual photon and we can consider the photon propagator $G_{\mu \nu}(k)$ in that situation instead of the polarization vector $\epsilon^{\mu}(k)$ of the photon which we considered in eq. (4.3) for the real photon emission from the electron only.

Hence if the photon is attached simultaneously to the electron of momentum $p^{\mu}$ and to the positron of momentum $q^{\mu}$ then the photon propagator $G^{\mu \nu}(k)$ can be split into the physical (transverse) propagator $G_{\text {phys }}^{\mu \nu}(k)$ and the pure gauge (longitudinal) propagator $G_{\text {pure }}^{\mu \nu}(k)$ as follows

$$
G^{\mu \nu}(k)=\frac{g^{\mu \nu}}{k^{2}}=G_{\mathrm{phys}}^{\mu \nu}(k)+G_{\mathrm{pure}}^{\mu \nu}(k)
$$

which is equivalent to eq. (4.3) of our paper where in the infrared (IR) limit (see eq. (2.1) of $[24])$

and

$$
G_{\text {phys }}^{\mu \nu}(k)=\frac{1}{k^{2}}\left[g^{\mu \nu}-\frac{p \cdot q}{(p \cdot k)(q \cdot k)} k^{\mu} k^{\nu}\right]
$$

$$
G_{\text {pure }}^{\mu \nu}(k)=\frac{p \cdot q}{(p \cdot k)(q \cdot k)} \frac{k^{\mu} k^{\nu}}{k^{2}} .
$$

For the virtual photon interacting simultaneously with the electron of momentum $p^{\mu}$ and the positron of momentum $q^{\mu}$ the eikonal Feynman rule for the infrared (IR) divergences is given by

(see, for example, eq. (29) of [20]).

$$
\frac{p^{\mu}}{p \cdot k} G_{\mu \nu}(k) \frac{q^{\nu}}{q \cdot k}
$$


Hence from eqs. (4.9), (4.10), (4.11) and (4.12) we find

$$
\frac{p^{\mu}}{p \cdot k} G_{\mu \nu}^{\text {pure }}(k) \frac{q^{\nu}}{q \cdot k} \rightarrow \infty, \quad \text { as } \quad k \rightarrow 0
$$

and

$$
\frac{p^{\mu}}{p \cdot k} G_{\mu \nu}^{\text {phys }}(k) \frac{q^{\nu}}{q \cdot k}=0 .
$$

The above equation is equivalent to eq. (4.7) of our paper. Hence one finds that the pure gauge part is the source of the infrared divergence.

It is well known that the light-like charge produces pure gauge field at all the timespace points $x^{\mu}$ except at the positions perpendicular to the direction of the motion of the charge at the time of closest approach in classical mechanics [25-27]. This is also true in quantum field theory which can be shown as follows.

The generating functional for the photon field $Q^{\mu}(x)$ in the presence of external source $J^{\mu}(x)$ is given by

$$
Z[J]=\int[d Q] e^{i \int d^{4} x\left[-\frac{1}{4}\left[\partial_{\mu} Q_{\nu}(x)-\partial_{\nu} Q_{\mu}(x)\right]\left[\partial^{\mu} Q^{\nu}(x)-\partial^{\nu} Q^{\mu}(x)\right]-\frac{1}{2 \alpha}\left(\partial_{\nu} Q^{\nu}\right)^{2}+J_{\mu}(x) Q^{\mu}(x)\right]}
$$

which gives the effective action $[1,28-30]$

$$
<0 \mid 0>_{J}=\frac{Z[J]}{Z[0]}=e^{i S_{\text {eff }}}, \quad S_{\text {eff }}=-\frac{1}{2} \int d^{4} x J_{\mu}(x) \frac{1}{\partial^{2}} J^{\mu}(x)=\int d^{4} x \mathcal{L}_{\text {eff }}(x)
$$

where $\mathcal{L}_{\text {eff }}(x)$ is the effective lagrangian density.

The eikonal current density $J_{\text {eik }}^{\mu}(x)$ of the charge with light-like four velocity $l^{\mu}$ can be obtained from the eikonal term of eq. (4.1)

$$
e \int \frac{d^{4} k}{(2 \pi)^{4}} \frac{l \cdot Q(k)}{l \cdot k+i \epsilon}=-i \int d^{4} x J_{\mathrm{eik}}^{\mu}(x) Q_{\mu}(x)
$$

which gives the light-like eikonal current density

$$
J_{\text {eik }}^{\mu}(x)=e \int_{0}^{\infty} d \lambda l^{\mu} \delta^{(4)}(x-\lambda l) .
$$

Using eq. (4.18) in (4.16) we find

$$
\mathcal{L}_{\text {eff }}^{\text {eik }}(x)=\frac{e^{2}}{2}\left[\frac{l^{2}}{(l \cdot x)^{2}}\right]^{2}=0, \quad \quad l^{2}=0, \quad x \cdot l \neq 0
$$

which means the eikonal current of light-like charge produces pure gauge field in quantum field theory at all the time-space positions $x^{\mu}$ except at the positions perpendicular to the direction of the motion of the charge at the time of closest approach $(l \cdot x \neq 0)$ which agrees with the corresponding result of the classical mechanics.

From eq. (4.1) the non-eikonal contribution

$$
e \gamma^{\mu} \gamma^{\nu} \int \frac{d^{4} k}{(2 \pi)^{4}} \frac{k_{\mu} Q_{\nu}(k)}{2 p \cdot k+i \epsilon}=\int d^{4} x J_{\text {noneik }}^{\mu}(x) Q_{\mu}(x)
$$


gives the non-eikonal current density

$$
J_{\text {noneik }}^{\mu}(x)=e \gamma^{\nu} \gamma^{\mu} \int_{0}^{\infty} d \lambda \partial_{\nu} \delta^{(4)}(x-\lambda p) .
$$

Using eqs. (4.21) and (4.18) in eq. (4.16) we find that the effective interaction lagrangian density due to the interaction between the (light-like or non-light-like) non-eikonal current and the gauge field generated by the light-like eikonal current is given by

$$
\mathcal{L}_{\text {eff }}^{\operatorname{int}}(x)=l^{2} \frac{e^{2}}{2} \frac{(p \cdot l)(p \cdot x)-p^{2} l \cdot x}{(l \cdot x)^{3}\left[(p \cdot x)^{2}-p^{2} x^{2}\right]^{\frac{3}{2}}}=0, \quad l^{2}=0, \quad x \cdot l \neq 0, \quad p \cdot x \neq 0
$$

which implies that the light-like eikonal line can be replaced by the pure gauge background field to study factorization of infrared divergences in quantum field theory without modifying the finite value of the physical cross section.

Hence we find that since the light-like eikonal current produces pure gauge field, the study of infrared divergence due to soft photon (eikonal photon) emission due to the presence of light-like eikonal line can be simplified in quantum field theory by using pure gauge without modifying the finite value of the cross section. Since the light-like eikonal current produces pure gauge field in classical mechanics and in quantum field theory, the factorization of infrared divergence due to the presence of light-like eikonal line can be studied by using path integral formulation of the background field method of quantum field theory in the presence of pure gauge background field [1, 31-33].

In QED the light-like electric charge produces $\mathrm{U}(1)$ pure gauge field $A^{\mu}(x)$ given by

$$
A_{\mu}(x)=\partial_{\mu} \omega(x)
$$

In QCD the light-like color charges produce $\mathrm{SU}(3)$ pure gauge field given by $[1,31,32]$

$$
T^{c} A^{\mu c}(x)=\frac{1}{i g}\left[\partial^{\mu} \Phi(x)\right] \Phi^{-1}(x)
$$

where $\Phi(x)$ is the gauge link given by eq. (1.6) where $l^{\mu}$ is the light-like four velocity.

\section{Proof of factorization of infrared divergence in color singlet $\mathrm{P}$-wave heavy quarkonium production}

As discussed above the factorization of infrared divergences due to the soft gluons emission/absorption with the light-like eikonal line can be studied by using the path integral formulation of the background field method of QCD $[1,31,32]$ in the presence of SU(3) pure gauge background field $A^{\mu a}(x)$ as given by eq. (4.24). We follow the notation of [34-36] and denote the quantum gluon field by $Q^{\mu a}$ and the background field by $A^{\mu a}$.

In the path integral formulation of QCD we find $[34,37]$

$$
\begin{aligned}
& <0\left|\bar{\Psi}\left(x^{\prime}\right) \bar{\nabla}_{x^{\prime}} \Psi\left(x^{\prime}\right) \cdot \bar{\Psi}\left(x^{\prime \prime}\right) \bar{\nabla}_{x^{\prime \prime}} \Psi\left(x^{\prime \prime}\right)\right| 0>= \\
& \quad \int \Pi_{l=1}^{3}\left[d \bar{\psi}_{l}\right]\left[d \psi_{l}\right][d \bar{\Psi}][d \Psi][d Q] \bar{\Psi}\left(x^{\prime}\right) \bar{\nabla}_{x^{\prime}} \Psi\left(x^{\prime}\right) \cdot \bar{\Psi}\left(x^{\prime \prime}\right) \bar{\nabla}_{x^{\prime \prime}} \Psi\left(x^{\prime \prime}\right) \operatorname{det}\left(\frac{\delta \partial_{\mu} Q^{\mu c}}{\delta \omega^{d}}\right) \\
& \quad e^{i \int d^{4} x\left[\sum_{l=1}^{3} \bar{\psi}_{l}\left[i \gamma^{\mu} \partial_{\mu}-m_{l}+g T^{a} \gamma^{\mu} Q_{\mu}^{a}\right] \psi_{l}+\bar{\Psi}\left[i \gamma^{\mu} \partial_{\mu}-M+g T^{a} \gamma^{\mu} Q_{\mu}^{a}\right] \Psi-\frac{1}{4} F_{\mu \nu}^{a 2}[Q]-\frac{1}{2 \alpha}\left(\partial_{\mu} Q^{\mu a}\right)^{2}\right]}
\end{aligned}
$$


and in the path integral formulation of the background field method of QCD we find [34-36]

$$
\begin{aligned}
<0\left|\bar{\Psi}\left(x^{\prime}\right) \bar{\nabla}_{x^{\prime}} \Psi\left(x^{\prime}\right) \cdot \bar{\Psi}\left(x^{\prime \prime}\right) \bar{\nabla}_{x^{\prime \prime}} \Psi\left(x^{\prime \prime}\right)\right| 0>_{A}= \\
\quad \int \Pi_{l=1}^{3}\left[d \bar{\psi}_{l}\right]\left[d \psi_{l}\right][d \bar{\Psi}][d \Psi][d Q] \bar{\Psi}\left(x^{\prime}\right) \bar{\nabla}_{x^{\prime}} \Psi\left(x^{\prime}\right) \cdot \bar{\Psi}\left(x^{\prime \prime}\right) \bar{\nabla}_{x^{\prime \prime}} \Psi\left(x^{\prime \prime}\right) \operatorname{det}\left(\frac{\delta G^{c}(Q)}{\delta \omega^{d}}\right) \\
e^{i \int d^{4} x\left[\sum_{l=1}^{3} \bar{\psi}_{l}\left[i \gamma^{\mu} \partial_{\mu}-m_{l}+g T^{a} \gamma^{\mu}(A+Q)_{\mu}^{a}\right] \psi_{l}+\bar{\Psi}\left[i \gamma^{\mu} \partial_{\mu}-M+g T^{a} \gamma^{\mu}(A+Q)_{\mu}^{a}\right] \Psi-\frac{1}{4} F_{\mu \nu}^{a 2}[A+Q]-\frac{1}{2 \alpha}\left(G^{a}(Q)\right)^{2}\right]}
\end{aligned}
$$

where

$$
F_{\mu \nu}^{c}[A+Q]=\partial_{\mu}\left[A_{\nu}^{c}+Q_{\nu}^{c}\right]-\partial_{\nu}\left[A_{\mu}^{c}+Q_{\mu}^{c}\right]+g f^{c b a}\left[A_{\mu}^{b}+Q_{\mu}^{b}\right]\left[A_{\nu}^{a}+Q_{\nu}^{a}\right], \quad F_{\mu \nu}^{2}=F_{\mu \nu} F^{\mu \nu}
$$

and

$$
G^{c}(Q)=\partial_{\mu} Q^{\mu c}+g f^{c b a} A_{\mu}^{b} Q^{\mu a}
$$

is the gauge fixing term and the type I gauge transformation in the background field method of QCD is given by

$$
\begin{aligned}
& T^{c} A^{\prime \mu c}=\Phi T^{c} A^{\mu c} \Phi^{-1}+\frac{1}{i g}\left(\partial^{\mu} \Phi\right) \Phi^{-1} \\
& T^{c} Q^{\prime \mu c}=\Phi T^{c} Q^{\mu c} \Phi^{-1} .
\end{aligned}
$$

In eqs. (5.1) and (5.2) the $\psi_{l}$ is the Dirac field of the light quark of flavor $l=1,2,3=u, d, s$ of mass $m_{l}$, the $\Psi$ is the Dirac field of the heavy quark of mass $M$ and $\bar{\nabla}$ is defined by eq. (1.4).

Changing the integration variable $Q \rightarrow Q-A$ inside the path integration in eq. (5.2) we find

$$
\begin{aligned}
< & 0\left|\bar{\Psi}\left(x^{\prime}\right) \bar{\nabla}_{x^{\prime}} \Psi\left(x^{\prime}\right) \cdot \bar{\Psi}\left(x^{\prime \prime}\right) \bar{\nabla}_{x^{\prime \prime}} \Psi\left(x^{\prime \prime}\right)\right| 0>_{A}= \\
& \int \Pi_{l=1}^{3}\left[d \bar{\psi}_{l}\right]\left[d \psi_{l}\right][d \bar{\Psi}][d \Psi][d Q] \bar{\Psi}\left(x^{\prime}\right) \bar{\nabla}_{x^{\prime}} \Psi\left(x^{\prime}\right) \cdot \bar{\Psi}\left(x^{\prime \prime}\right) \bar{\nabla}_{x^{\prime \prime}} \Psi\left(x^{\prime \prime}\right) \operatorname{det}\left(\frac{\delta G_{f}^{c}(Q)}{\delta \omega^{d}}\right) \\
& e^{i \int d^{4} x\left[\sum_{l=1}^{3} \bar{\psi}_{l}\left[i \gamma^{\mu} \partial_{\mu}-m_{l}+g T^{a} \gamma^{\mu} Q_{\mu}^{a}\right] \psi_{l}+\bar{\Psi}\left[i \gamma^{\mu} \partial_{\mu}-M+g T^{a} \gamma^{\mu} Q_{\mu}^{a}\right] \Psi-\frac{1}{4} F_{\mu \nu}^{a 2}[Q]-\frac{1}{2 \alpha}\left(G_{f}^{a}(Q)\right)^{2}\right]}
\end{aligned}
$$

where

$$
G_{f}^{c}(Q)=\partial_{\mu} Q^{\mu c}+g f^{c b a} A_{\mu}^{b} Q^{\mu a}-\partial_{\mu} A^{\mu c}
$$

and eq. (5.5) becomes

$$
T^{c} Q^{\prime \mu c}=\Phi T^{c} Q^{\mu c} \Phi^{-1}+\frac{1}{i g}\left(\partial^{\mu} \Phi\right) \Phi^{-1} .
$$

Since the change of integration variables from unprimed variables to primed variables does not change the value of the integration we find from eq. (5.6)

$$
\begin{aligned}
& <0\left|\bar{\Psi}\left(x^{\prime}\right) \bar{\nabla}_{x^{\prime}} \Psi\left(x^{\prime}\right) \cdot \bar{\Psi}\left(x^{\prime \prime}\right) \bar{\nabla}_{x^{\prime \prime}} \Psi\left(x^{\prime \prime}\right)\right| 0>_{A}= \\
& \int \Pi_{l=1}^{3}\left[d \bar{\psi}_{l}^{\prime}\right]\left[d \psi_{l}^{\prime}\right]\left[d \bar{\Psi}^{\prime}\right]\left[d \Psi^{\prime}\right]\left[d Q^{\prime}\right] \bar{\Psi}^{\prime}\left(x^{\prime}\right) \bar{\nabla}_{x^{\prime}} \Psi^{\prime}\left(x^{\prime}\right) \cdot \bar{\Psi}^{\prime}\left(x^{\prime \prime}\right) \bar{\nabla}_{x^{\prime \prime}} \Psi^{\prime}\left(x^{\prime \prime}\right) \operatorname{det}\left(\frac{\delta G^{c}\left(Q^{\prime}\right)}{\delta \omega^{d}}\right) \\
& e^{i \int d^{4} x\left[\sum_{l=1}^{3} \bar{\psi}_{l}^{\prime}\left[i \gamma^{\mu} \partial_{\mu}-m_{l}+g T^{a} \gamma^{\mu} Q_{\mu}^{\prime a}\right] \psi_{l}^{\prime}+\bar{\Psi}^{\prime}\left[i \gamma^{\mu} \partial_{\mu}-M+g T^{a} \gamma^{\mu} Q_{\mu}^{\prime a}\right] \Psi^{\prime}-\frac{1}{4} F^{a}{ }_{\mu \nu}^{2}\left[Q^{\prime}\right]-\frac{1}{2 \alpha}\left(G_{f}^{a}\left(Q^{\prime}\right)\right)^{2}\right]} \text {. }
\end{aligned}
$$


From

$$
\psi^{\prime}=\Phi \psi
$$

and from eq. (5.8) we find $[1,31,32]$

$$
\begin{aligned}
{\left[d Q^{\prime}\right]=[d Q], \quad\left[d \bar{\psi}_{l}^{\prime}\right]\left[d \psi_{l}^{\prime}\right] } & =\left[d \bar{\psi}_{l}\right]\left[d \psi_{l}\right], \quad\left[d \bar{\Psi}^{\prime}\right]\left[d \Psi^{\prime}\right]=[d \bar{\Psi}][d \Psi], \\
\bar{\psi}_{l}^{\prime}\left[i \gamma^{\mu} \partial_{\mu}-m_{l}+g T^{c} \gamma^{\mu} Q_{\mu}^{\prime c}\right] \psi_{l}^{\prime} & =\bar{\psi}_{l}\left[i \gamma^{\mu} \partial_{\mu}-m_{l}+g T^{c} \gamma^{\mu} Q_{\mu}^{c}\right] \psi_{l}, \\
\bar{\Psi}^{\prime}\left[i \gamma^{\mu} \partial_{\mu}-M+g T^{c} \gamma^{\mu} Q_{\mu}^{\prime c}\right] \Psi^{\prime} & =\bar{\Psi}\left[i \gamma^{\mu} \partial_{\mu}-M+g T^{c} \gamma^{\mu} Q_{\mu}^{c}\right] \Psi, \quad F^{2}\left[Q^{\prime}\right]=F^{2}[Q] \\
\left(G_{f}^{c}\left(Q^{\prime}\right)\right)^{2} & =\left(\partial_{\mu} Q^{\mu c}\right)^{2}, \quad \operatorname{det}\left[\frac{\delta G_{f}^{c}\left(Q^{\prime}\right)}{\delta \omega^{d}}\right]=\operatorname{det}\left[\frac{\delta\left(\partial_{\mu} Q^{\mu c}\right)}{\delta \omega^{d}}\right] .
\end{aligned}
$$

From eqs. (5.11), (5.10), (5.9) and (5.1) we find

$$
\begin{aligned}
& <0\left|\bar{\Psi}\left(x^{\prime}\right) \bar{\nabla}_{x^{\prime}} \Psi\left(x^{\prime}\right) a_{H}^{\dagger} \cdot a_{H} \bar{\Psi}(x) \bar{\nabla}_{x} \Psi(x)\right| 0> \\
& =<0\left|\bar{\Psi}\left(x^{\prime}\right) \Phi\left(x^{\prime}\right) \bar{\nabla}_{x^{\prime}} \Phi^{\dagger}\left(x^{\prime}\right) \Psi\left(x^{\prime}\right) a_{H}^{\dagger} \cdot a_{H} \bar{\Psi}(x) \Phi(x) \bar{\nabla}_{x} \Phi^{\dagger}(x) \Psi(x)\right| 0>_{A}
\end{aligned}
$$

which proves factorization of infrared divergences in color singlet $\mathrm{P}$-wave heavy quarkonium production at all order in coupling constant in QCD where the gauge link $\Phi(x)$ is given by eq. (1.6). Note that the $\bar{\nabla}$ in the left hand side of eq. (5.12) is defined by eq. (1.4) and the $\bar{\nabla}$ in the right hand side of eq. (5.12) is defined by eq. (1.7).

\section{Correct definition of non-perturbative matrix element of color singlet $\mathrm{P}$-wave heavy quarkonium production}

From eq. (5.12) we find that the correct definition of the gauge invariant color singlet $\mathrm{P}$-wave non-perturbative matrix element of heavy quarkonium production which is consistent with factorization of infrared divergences in QCD at all orders in coupling constant is given by

$$
<0\left|\mathcal{O}_{H}\right| 0>=<0\left|\chi^{\dagger} \Phi \bar{\nabla} \Phi^{\dagger} \xi a_{H}^{\dagger} \cdot a_{H} \xi^{\dagger} \Phi \bar{\nabla} \Phi^{\dagger} \chi\right| 0>
$$

which reproduces eq. (1.5) where the gauge link $\Phi(x)$ is given by eq. (1.6) and $\bar{\nabla}$ is defined in eq. (1.7).

Since the left hand side of eq. (5.12) is independent of the light-like vector $l^{\mu}$ one finds that the right hand side of eq. (5.12) is independent of the light-like vector $l^{\mu}$ used to define the gauge link $\Phi(x)$ in eq. (1.6). This proves that the long-distance behavior of the color singlet P-wave non-perturbative matrix element $<0\left|\chi^{\dagger} \Phi \bar{\nabla} \Phi^{\dagger} \xi a_{H}^{\dagger} \cdot a_{H} \xi^{\dagger} \Phi \bar{\nabla} \Phi^{\dagger} \chi\right| 0>$ in eq. (6.1) is independent of the light-like vector $l^{\mu}$ at all order in coupling constant in QCD.

This completes the derivation of the correct definition of the non-perturbative matrix element of color singlet P-wave heavy quarkonium production which is gauge invariant and is consistent with the factorization of infrared divergences at all order in coupling constant in QCD.

Note that the relationship between the color singlet P-wave long distance matrix element (LDME) and the derivative of the non-relativistic wave function at origin $\left|R^{\prime}(0)\right|^{2}$ 
is very useful because it helps us to reduce the number of non-perturbative input parameters to be determined. Hence it is important to discuss how this relationship is affected by the redefinition in eq. (1.5). First of all it is found that if one uses the derivative of the non-relativistic wave function at the origin $\left|R^{\prime}(0)\right|^{2}$ in eq. (1.1) then the infrared (IR) divergences do not cancel $[8,10-14]$, i.e., the non-perturbative effects can not be factored into the derivative of the non-relativistic wave function at the origin $\left|R^{\prime}(0)\right|^{2}$. In order to cancel the infrared (IR) divergences and to be consistent with the factorization theorem at all orders in coupling constant the definition of the color singlet P-wave non-perturbative matrix element from eq. (1.5) should be used in eq. (1.1). Note that since the color singlet $\mathrm{P}$-wave non-perturbative matrix element in eq. (1.5) is a non-perturbative quantity it can not be studied by using perturbative QCD. Due to these infrared (IR) divergences, factorization and non-perturbative QCD issues there is no simple relation between the color singlet $\mathrm{P}$-wave non-perturbative matrix element in eq. (1.5) at all orders in coupling constant and the derivative of the non-relativistic wave function at the origin $\left|R^{\prime}(0)\right|^{2}$. The color singlet $\mathrm{P}$-wave non-perturbative matrix element in eq. (1.5) at all orders in coupling constant can be studied by using the non-perturbative QCD. In the phenomenological studies of the infrared sensitive processes in color singlet P-wave heavy quarkonium production, the definition from eq. (1.5) should be used in eq. (1.1) to extract the non-perturbative matrix element from the experiments in order to be consistent with the factorization of the infrared (IR) divergences at all orders in coupling constant.

\section{Conclusions}

Recently we have proved factorization of infrared divergences in NRQCD S-wave heavy quarkonium production at high energy colliders at all orders in coupling constant. One of the problem which still exists in the higher order pQCD calculation of color singlet Pwave heavy quarkonium production/anihillation is the appearance of non-canceling infrared divergences due to real soft gluons exchange, although no such infrared divergences are present in the color singlet S-wave heavy quarkonium.

In this paper we have found that since the non-perturbative matrix element of the color singlet $\mathrm{P}$-wave heavy quarkonium production contains derivative operators, the gauge links are necessary to make it gauge invariant and be consistent with the factorization of such non-canceling infrared divergences at all orders in coupling constant. In case of color singlet S-wave heavy quarkonium production the gauge links cancel because of the absence of derivative operators which is consistent with the absence of such non-canceling infrared divergences at all orders in coupling constant.

Open Access. This article is distributed under the terms of the Creative Commons Attribution License (CC-BY 4.0), which permits any use, distribution and reproduction in any medium, provided the original author(s) and source are credited. 


\section{References}

[1] G.C. Nayak, Proof of NRQCD factorization at all orders in the coupling constant in heavy quarkonium production, Eur. Phys. J. C 76 (2016) 448 [arXiv:1506.02593] [INSPIRE].

[2] E598 collaboration, J.J. Aubert et al., Experimental Observation of a Heavy Particle J, Phys. Rev. Lett. 33 (1974) 1404 [InSPIRE].

[3] SLAC-SP-017 collaboration, J.E. Augustin et al., Discovery of a Narrow Resonance in $e^{+} e^{-}$ Annihilation, Phys. Rev. Lett. 33 (1974) 1406 [Adv. Exp. Phys. 5 (1976) 141] [InSPIRE].

[4] T. Appelquist and H.D. Politzer, Orthocharmonium and $e^{+} e^{-}$Annihilation, Phys. Rev. Lett. 34 (1975) 43 [INSPIRE].

[5] T. Appelquist and H.D. Politzer, Heavy Quarks and Longlived Hadrons, Phys. Rev. D 12 (1975) 1404 [INSPIRE].

[6] D.J. Gross and F. Wilczek, Ultraviolet Behavior of Nonabelian Gauge Theories, Phys. Rev. Lett. 30 (1973) 1343 [INSPIRE].

[7] H.D. Politzer, Reliable Perturbative Results for Strong Interactions?, Phys. Rev. Lett. 30 (1973) 1346 [INSPIRE].

[8] A. Petrelli, M. Cacciari, M. Greco, F. Maltoni and M.L. Mangano, NLO production and decay of quarkonium, Nucl. Phys. B 514 (1998) 245 [hep-ph/9707223] [INSPIRE].

[9] R. Barbieri, E. d'Emilio, G. Curci and E. Remiddi, Strong Radiative Corrections to Annihilations of Quarkonia in QCD, Nucl. Phys. B 154 (1979) 535 [InSPIRE].

[10] R. Barbieri, R. Gatto and E. Remiddi, Singular Binding Dependence in the Hadronic Widths of $1++$ and 1+- Heavy Quark anti-Quark Bound States, Phys. Lett. 61B (1976) 465 [INSPIRE].

[11] R. Barbieri, M. Caffo and E. Remiddi, Gluon Jets From Quarkonia, Nucl. Phys. B 162 (1980) 220 [inSPIRE].

[12] R. Barbieri, M. Caffo, R. Gatto and E. Remiddi, Strong QCD Corrections to $p$ Wave Quarkonium Decays, Phys. Lett. 95B (1980) 93 [INSPIRE].

[13] R. Barbieri, M. Caffo, R. Gatto and E. Remiddi, QCD corrections to P-wave quarkonium decays, Nucl. Phys. B 192 (1981) 61 [INSPIRE].

[14] W. Kwong, P.B. Mackenzie, R. Rosenfeld and J.L. Rosner, Quarkonium Annihilation Rates, Phys. Rev. D 37 (1988) 3210 [INSPIRE].

[15] I. Harris and L.M. Brown, Radiative Corrections to Pair Annihilation, Phys. Rev. 105 (1957) 1656 [INSPIRE].

[16] P.B. Mackenzie and G.P. Lepage, QCD Corrections to the Gluonic Width of the Upsilon Meson, Phys. Rev. Lett. 47 (1981) 1244 [InSPIRE].

[17] G.T. Bodwin, E. Braaten and G.P. Lepage, Rigorous QCD analysis of inclusive annihilation and production of heavy quarkonium, Phys. Rev. D 51 (1995) 1125 [Erratum ibid. D 55 (1997) 5853] [hep-ph/9407339] [INSPIRE].

[18] G.C. Nayak, J.-W. Qiu and G.F. Sterman, Fragmentation, factorization and infrared poles in heavy quarkonium production, Phys. Lett. B 613 (2005) 45 [hep-ph/0501235] [INSPIRE].

[19] G.C. Nayak, J.-W. Qiu and G.F. Sterman, Fragmentation, NRQCD and NNLO factorization analysis in heavy quarkonium production, Phys. Rev. D 72 (2005) 114012 [hep-ph/0509021] [INSPIRE]. 
[20] G.C. Nayak, J.-W. Qiu and G.F. Sterman, NRQCD Factorization and Velocity-dependence of NNLO Poles in Heavy Quarkonium Production, Phys. Rev. D 74 (2006) 074007 [hep-ph/0608066] [INSPIRE].

[21] G.A. Schuler, Quarkonium production and decays, hep-ph/9403387 [INSPIRE].

[22] E. Braaten and Y.-Q. Chen, Calculation of $P$ wave charmonium decay rates using dimensional regularization, Phys. Rev. D 55 (1997) 7152 [hep-ph/9701242] [INSPIRE].

[23] T. Matsui and H. Satz, $J / \psi$ Suppression by quark-gluon Plasma Formation, Phys. Lett. B 178 (1986) 416 [inSPIRE].

[24] G. Grammer, Jr. and D.R. Yennie, Improved treatment for the infrared divergence problem in quantum electrodynamics, Phys. Rev. D 8 (1973) 4332 [InSPIRE].

[25] J.C. Collins, D.E. Soper and G.F. Sterman, Factorization for Short Distance Hadron-Hadron Scattering, Nucl. Phys. B 261 (1985) 104 [INSPIRE].

[26] G.C. Nayak, General Form of the Color Potential Produced by Color Charges of the Quark, JHEP 03 (2013) 001 [arXiv:1201.2666] [INSPIRE].

[27] G.C. Nayak, General Form of Color Charge of the Quark, Eur. Phys. J. C 73 (2013) 2442 [arXiv: 1201.2672] [INSPIRE].

[28] G.C. Nayak and P. van Nieuwenhuizen, Soft-gluon production due to a gluon loop in a constant chromo-electric background field, Phys. Rev. D 71 (2005) 125001 [hep-ph/0504070] [INSPIRE].

[29] G.C. Nayak, Non-perturbative quark-antiquark production from a constant chromo-electric field via the Schwinger mechanism, Phys. Rev. D 72 (2005) 125010 [hep-ph/0510052] [INSPIRE].

[30] F. Cooper and G.C. Nayak, Non-perturbative gluon pair production from a constant chromo-electric field via the Schwinger mechanism in arbitrary gauge, Phys. Rev. D 73 (2006) 065005 [hep-ph/0511053] [INSPIRE].

[31] G.C. Nayak, Light-Like Wilson Line in QCD Without Path Ordering, Phys. Part. Nucl. Lett. 13 (2016) 417 [arXiv:1506.07146] [InSPIRE].

[32] G.C. Nayak, Jet Quenching and Gluon to Hadron Fragmentation Function in Non-Equilibrium QCD at RHIC and LHC, Phys. Part. Nucl. Lett. 14 (2017) 18 [arXiv: 1509.01148] [INSPIRE].

[33] R. Tucci, Factorization of Soft and Collinear Divergences in QCD in Feynman Gauge via Background Field Gauge, Phys. Rev. D 32 (1985) 945 [Erratum ibid. D 34 (1986) 1235] [INSPIRE].

[34] L.F. Abbott, The Background Field Method Beyond One Loop, Nucl. Phys. B 185 (1981) 189 [inSPIRE].

[35] G. 't Hooft, An algorithm for the poles at dimension four in the dimensional regularization procedure, Nucl. Phys. B 62 (1973) 444 [INSPIRE].

[36] H. Kluberg-Stern and J.B. Zuber, Renormalization of Nonabelian Gauge Theories in a Background Field Gauge. 1. Green Functions, Phys. Rev. D 12 (1975) 482 [InSPIRE].

[37] T. Muta, World Scientific Lecture nNtes in Physics. Vol. 5: Foundations of Quantum Chromodynamics, World Scientific, New York U.S.A. (1987). 